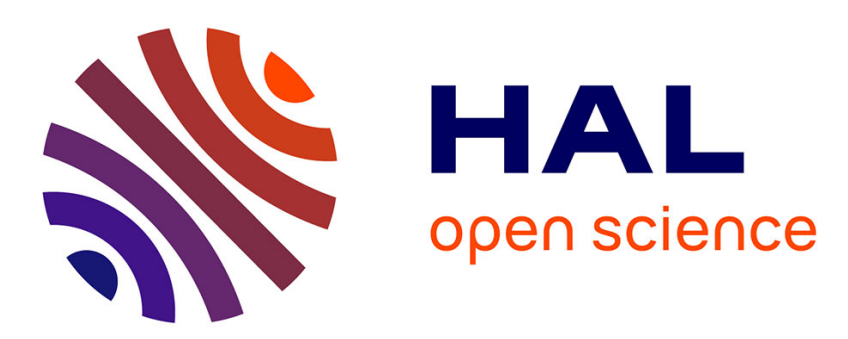

\title{
X-ray standing waves on the surface of the crystal with the uniform strain gradient
}

I. Vartanyantz, M. Kovalchuk, I. Ustimenko

\section{To cite this version:}

I. Vartanyantz, M. Kovalchuk, I. Ustimenko. X-ray standing waves on the surface of the crystal with the uniform strain gradient. Journal de Physique IV Proceedings, 1994, 04 (C9), pp.C9-191-C9-194. 10.1051/jp4:1994933 . jpa-00253494

\section{HAL Id: jpa-00253494 https://hal.science/jpa-00253494}

Submitted on 1 Jan 1994

HAL is a multi-disciplinary open access archive for the deposit and dissemination of scientific research documents, whether they are published or not. The documents may come from teaching and research institutions in France or abroad, or from public or private research centers.
L'archive ouverte pluridisciplinaire HAL, est destinée au dépôt et à la diffusion de documents scientifiques de niveau recherche, publiés ou non, émanant des établissements d'enseignement et de recherche français ou étrangers, des laboratoires publics ou privés. 


\title{
X-ray standing waves on the surface of the crystal with the uniform strain gradient
}

I.A. Vartanyantz, M.V. Kovalchuk and I.N. Ustimenko

Institute of Crystallography RAS, Leninsky pr. 59, 117333 Moscow, Russia

\begin{abstract}
The results of theoretical investigation of X-ray Standing Waves (XSW) on the surface of the crystal with the Uniform Strain Gradient (USG) are presented. Bent crystals are the typical examples of the crystals with the USG. The proposed theory is based on the Takagi-Taupin theory of X-ray propagation in a weakly deformed crystal. This theory is applied for calculation of the angular dependencies of reflection coefficient and XSW on the surface of the crystal with USG for the specific case of $\mathrm{Si}(400)$ thick crystal, CuKa radiation. The behaviour of the XSW with the different position of adsorbed atoms (different values of the phase shift) are analyzed.
\end{abstract}

\section{INTRODUCTION}

The X-ray Standing Wave (XSW) method considered for the first time for the perfect crystal by Batterman [1,2], nowadays is a powerful tool for investigation of the structure of real crystals [3] and the position of adsorbed atoms on the surface of the crystals [4]. In most of applications crystals used as a substrates for XSW experiments are perfect crystals. However it would be quite interesting to investigate the influence of deformation on the XSW pattern on the surface of the deformed crystal. Theory of XSW method when the deformation field $\mathbf{u}(\mathbf{r})$ depends only on the depth $\mathrm{z}$ in the crystal and the incident wave is a plane wave was elaborated in [5]. However for a large class of crystals with a Uniform Strain Gradient (USG) (in this case the strain field is a two dimensional function) the behaviour of the XSW field on the surface of the crystal have not been investigated. At the same time investigation of crystals with a USG by different diffraction methods is of great physical and practical point of view. Bent crystals, widely used as X-ray monochromators at synchrotron radiation sources [6] and as X-ray microscopes and focusing spectrometers for plasma analysis [7], are the typical examples of the crystals with the USG.

In previous papers [8,9] results of the theoretical treatment of the XSW method and the secondary radiation yield (fluorescence and photoemission yield) in a bent crystal were presented. This results were obtained in the frame of Taupin approach [10]. This approach gives the possibility for the cylindrically bent crystal and the special incident wave, to reduce the general problem of two variables to the problem of one variable (the so-called Taupin problem). However, this situation is hardly realized in practice.

In this paper more realistic approach based on the Takagi-Taupin theory [10-12] of X-ray propagation in a weakly deformed crystals with a USG is presented. The main principles of the theory of XSW method in such crystals are shortly developed in section 2 . This theory is applied for the analysis of the behaviour of the intensity of standing waves on the surface of the crystal with a USG (section 3 ). 


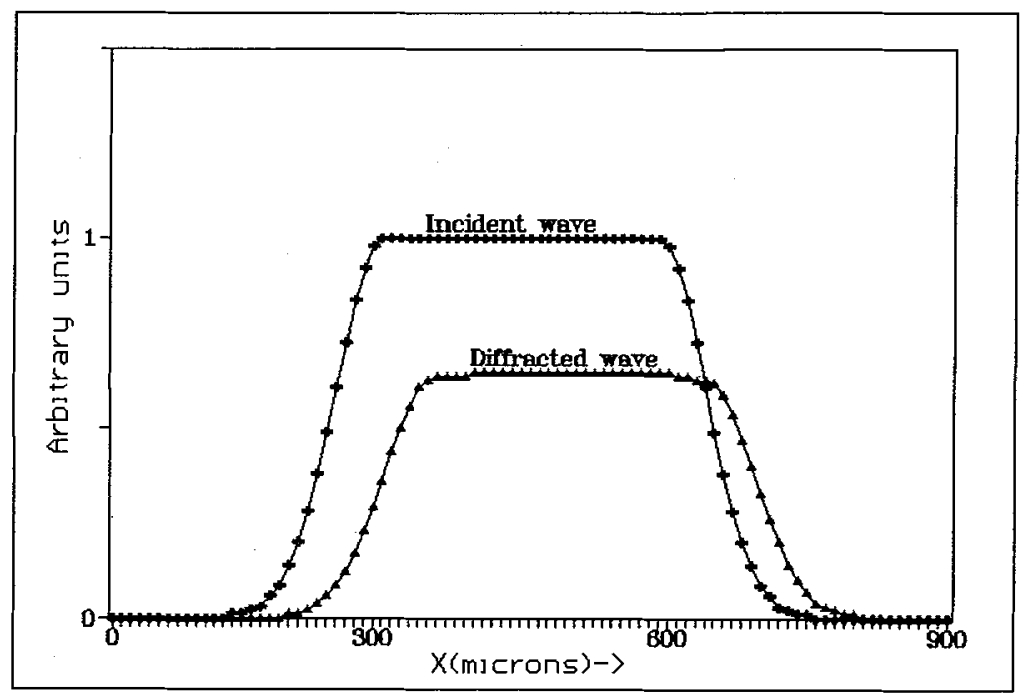

Fig. 1. Intensity distribution of the incident and diffracted beams along the crystal surface. The diffracted beam was calculated for the crystal with the USG and the incidence angular deviation parameter $\mathrm{Y}=\mathbf{- 5}$.

\section{THEORY}

The X-ray amplitude of the total wavefield in the weakly deformed crystal in the two wave approximation is the coherent superposition of the incident $\mathbf{E}_{0}(\mathbf{r})$ and the diffracted $\mathbf{E}_{\mathrm{h}}(\mathbf{r})$ beams and is given by

$$
\mathbf{E}(\mathbf{r})=\mathbf{E}_{\mathrm{o}}(\mathbf{r}) \exp \left(\mathbf{i k}_{\mathrm{o}} \mathbf{r}\right)+\mathbf{E}_{\mathrm{h}}(\mathbf{r}) \exp \left(\mathbf{i k}_{\mathrm{h}} \mathbf{r}\right)
$$

where $\mathbf{k}_{0}$ and $\mathbf{k}_{h}=\mathbf{k}_{0}+\mathbf{h}$ are correspondingly incident and diffracted wave vectors and $\mathbf{h}$ is the reciprocal lattice vector.

In the general case of the weakly deformed crystal with an arbitrary deformation field, according to Takagi-Taupin theory [10-12], the amplitudes $\mathbf{E}_{\mathrm{o}}(\mathbf{r})$ and $\mathbf{E}_{\mathrm{h}}(\mathbf{r})$ are slowly varying functions of coordinate. They satisfy the Takagi-Taupin equations [10-12], which are derived directly from the Maxwell equations for the X-ray wave propagation in a crystal. This set of equations must be completed by the boundary conditions, which in the Bragg case of diffraction and thick crystal are

$$
\left.\mathbf{E}_{\mathrm{o}}(\mathbf{r})\right|_{\mathrm{z}=\mathrm{o}}=\mathbf{E}_{\mathrm{o}} \text { in }(\mathbf{r}) ;\left.\mathbf{E}_{\mathrm{h}}(\mathbf{r})\right|_{\mathrm{z}->\infty}=0 \text {. }
$$

The propagation of the X-ray wavefield in the deformed crystal is determined not by the displacement field $\mathbf{u}(\mathbf{r})$ itself, but by the second derivative of the projection of the displacement field $\mathbf{u}(\mathbf{r})$ on the diffraction vector $\mathbf{h}$ [13-15]. If this field is such that

$$
\partial^{2}(\mathbf{h u}(\mathbf{r})) / \partial \mathrm{s}_{\mathrm{o}} \partial \mathrm{s}_{\mathrm{h}}=\mathrm{B}
$$

where $s_{\mathrm{O}}$ and $\mathrm{s}_{\mathrm{h}}$ are coordinates in the direction of the incident $\mathbf{k}_{\mathrm{o}}$ and reflected wave vectors $\mathbf{k}_{h}$ and $B$ is a constant, then such deformation is called a USG (for e.g. this is the case of elastically bent crystal).

Rigorous solutions of the Takagi-Taupin equations with the deformation field (3) in the Bragg case of diffraction where obtained in [13] and analyzed in their asymptotic form in [14]. However for practical applications a much more convenient method is to integrate the TakagiTaupin equations in a numerical way.

The intensity of X-ray standing waves on the surface of the deformed crystal, according to (1), can be presented in the form

$$
\mathbf{I}(\Delta \theta)=\left.\left|\mathbf{E}_{\mathrm{o}}(\Delta \theta, \mathbf{r})+\mathbf{E}_{\mathrm{h}}(\Delta \theta, \mathbf{r}) \exp (\mathrm{i} \Delta \varphi)\right|^{2}\right|_{\mathrm{z}=\mathrm{o}}
$$

where $\Delta \theta$ is the angular deviation parameter and $\Delta \varphi$ is the phaseshift that is determined by the position of the impurity atoms on the surface of the crystal relative to diffraction planes.

As it follows from (4) the angular dependence of the X-ray standing wave on the surface of the crystal is determined mainly by two factors: the angular dependence of the amplitudes $\mathbf{E}_{\mathrm{o}}(\mathbf{r})$ and

$\mathbf{E}_{\mathrm{h}}(\mathbf{r})$

on the surface 


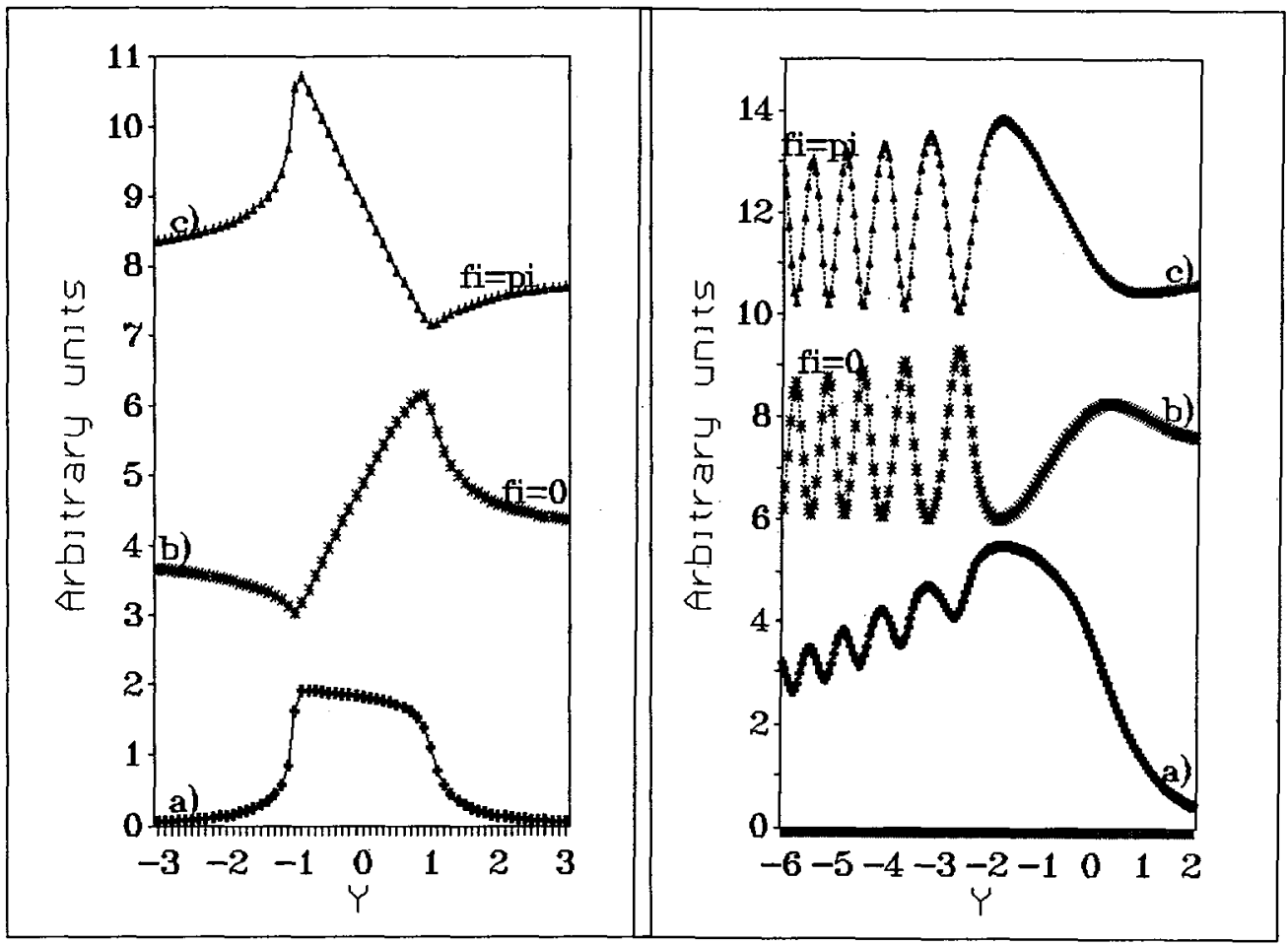

Fig. 2. The curves of the angular dependence of the X-ray reflection coefficient (a) and the X-ray standing waves (b and $c$ ) calculated on the surface of the perfect Si (400) crystal. The XSW curves correspond to different position (phaseshift $\Delta \varphi$ ) of the impurity atoms on the surface of the crystal. Points are results of numerical calculations, solid lines are results of the dynamical theory. $Y$ is a normalized angular deviation parameter.
Fig. 3. The curves of the angular dependence of the X-ray reflection coefficient (a) and the X-ray standing waves ( $b$ and c) calculated on the surface of the $\mathrm{Si}(400)$ crystal with a USG $\left(\mathrm{B}=2 \cos ^{2} \theta_{\mathrm{B}}\left(1 / \Lambda_{\mathrm{o}}\right) \beta_{\mathrm{c}}\right)$. The $\mathrm{XSW}$ curves correspond to different position (phaseshift $\Delta \varphi$ ) of the impurity atoms on the surface of the crystal.

of the crystal that is specified by the value of deformation. (in our case of the crystal with the USG by the constant B) and the position of impurity atoms (the phase $\Delta \varphi$ ).

The values of the amplitudes $\mathbf{E}_{0}(\mathbf{r})$ and $\mathbf{E}_{h}(\mathbf{r})$ on the surface of the crystal with the deformation field (3) were obtained from numerical integration of Takagi-Taupin equations. The algorithm for this calculations for Bragg case of diffraction is presented in details in [15].

\section{RESULTS AND DISCUSSION}

The curves of Angular Dependence (AD) of the X-ray Reflection Coefficient (XRC) $P_{R}=\left.\left|E_{h}(\mathbf{r}) / \mathbf{E}_{o}(\mathbf{r})\right|^{2}\right|_{z=0}$ and of the intensity of the standing wave (4) on the surface of the crystal with the USG were calculated for a specific case of (400) symmetrical Bragg diffraction, CuKa, s-polarized radiation in a thick crystal of silicon. The incident wave was taken in the "quasiplane" form (Fig.1) with a width $\sigma=400 \mu \mathrm{m}$ enough to reduce the influence of the boundary conditions. The diffracted beam for the fixed angular deviation $\Delta \theta$ is also shown on Fig. 1.

The results of numerical integration of Takagi-Taupin equations are presented on Fig. 2 and Fig. 3. The XSW curves on the surface of the crystal were calculated for two opposite situations: a) when the position of the impurity atoms just coincide with the position of the diffraction planes (the phase $\Delta \varphi=0$ curves b); b) when the position of the atoms is between the diffraction planes (the phase $\Delta \varphi=\pi$ curves c). 
Figure 2 presents the results of calculations in the case of the perfect crystal (constant $B=0$ ). Results obtained from numerical calculation of Takagi-Taupin equations (points on curves a-c) coincide exactly with the curves of $\mathrm{AD}$ (solid lines) obtained directly from the dynamical theory [2] for the perfect crystal. This results confirms the correctness of our approach as for the choice of the incident wave so as for the algorithm of calculations.

The results of calculations for deformed crystal are presented on Fig.3. The constant B was taken equal to $\mathrm{B}=2 \cos ^{2} \theta_{\mathrm{B}}\left(1 / \Lambda_{\mathrm{O}}\right) \boldsymbol{\beta}_{\mathrm{c}}$, where $\beta_{\mathrm{C}}=\pi /\left(2 \Lambda_{\mathrm{o}}\right)$ is the critical value usual in the theory of $X$-ray propagation in bent crystals [14,15], $\theta_{\mathrm{B}}$ is the Bragg angle and $\Lambda_{\mathrm{o}}$ is the extinction length in the symmetric Laue case. This value of constant $B$ correspond to $C=1$ from refs. $[8,9]$.

The curve of XRC (Fig. 3a) has a typical behaviour (the broadening in the central part and oscillations on the tails) obtained for the first time in [10]. The XSW curves (Fig. 3b,c) in the deformed crystal comparing to that in the perfect one are also broadened in the central part (in the so-called phase sensitive region) and have a sharp oscillations on the tails that correspond to oscillations on the XRC curve. The angular position of this oscillations depend not only from the value of deformation (determined in our case by the constant $B$ ) but also by the phase shift $\Delta \varphi$. The origin of this oscillations is the waveguide character of the X-ray propagation in the crystal with the USG $[10,14,9]$.

In the end we would like to note that results obtained in this paper on the basis of the Takagi-Taupin theory of X-ray propagation in the crystal with the USG are in a good agreement with the results of the previous papers [8,9] obtained in the frame of Taupin approach and a simple model of the curved crystal.

\section{Acknowledgements}

One of the authors (I.A.V.) wish to thank Dr. V.M.Kaganer for fruitful discussions.

\section{References}

[1] Batterman B.W., Phys. Rev. 133 (1964) A759-A764.

[2] Batterman B.W. and Cole H., Rev. Mod. Phys. 36 (1964) 681-764.

[3] Kovalchuk M.V. and Kohn V.G., Usp. Fiz. Nauk 149 (1986) 69-103 (Engl. transl. Sov. Phys.Usp. 29 (1986) 426).

[4] Zegenhagen J., Surface Science Reports 18 (1993) 199-271.

[5] Afanas'ev A.M. and Kohn V.G., Zh. Eksp. Teor. Fiz. 74 (1978) 300-313 (Engl. transl. Sov. Phys.-JETP 47 (1978) 154).

[6] Caciuffo R., Melone S., Rustichelli F. and Boeuf A., Phys. Rep. 152 (1987) 1-71.

[7] Uschmann I., Forster E., Gabel K., Holzer G. and Ensslen M., J. Appl. Cryst. 26 (1993) 405412.

[8] Vartanyantz I.A., Kovalchuk M.V., Beresovsky V.M., Phys. Stat Sol._135 (1993) 513-517.

[9] Vartanyantz I.A., Kovalchuk M.V., Beresovsky V.M., J.Phys. D: Appl. Phys._26 (1993) A197A201.

[10] Taupin D., Bull.Soc.Fr.Mineral.Crystallogr._87 (1964) 469-511.

[11] Takagi S., Acta Crystallogr._15 (1962) 1311-1312.

[12] Takagi S., J. Phys. Soc. Japan_26 (1969) 1239-1253.

[13] Chukhovskii F., Gabrielyan K. and Petrashen P.V., Acta Crystallogr._A34 (1978) 610-620.

[14] Chukhovskii F. and Malgrange C., Acta Crystallogr._A45 (1989) 732-738.

[15] Gronkowski J., Physics Reports 206 (1991) 1-41. 